\title{
Patient-reported outcome measures in community mental health teams: pragmatic evaluation of PHQ-9, GAD-7 and SWEMWBS
}

\author{
Paul Blenkiron, ${ }^{1}$ Lucy Goldsmith ${ }^{2}$
}

BJPsych Bulletin (2019) 43, 221-227, doi:10.1192/bjb.2019.20

${ }^{1}$ Tees, Esk and Wear Valleys NHS Foundation Trust, UK; ${ }^{2}$ St George's, University of London, UK

Correspondence to Paul Blenkiron (paul.blenkiron@nhs.net)

First received 27 Jul 2018, final revision 8 Feb 2019, accepted 19 Feb 2019

(C) The Authors 2019. This is an Open Access article, distributed under the terms of the Creative Commons Attribution licence (http:// creativecommons.org/licenses/by/ 4.0/), which permits unrestricted reuse, distribution, and reproduction in any medium, provided the original work is properly cited.

\begin{abstract}
Aims and method We evaluated routine use, acceptability and response rates for the Patient Health Questionnaire (PHQ-9), Generalised Anxiety Disorder Scale (GAD-7) and Short Warwick-Edinburgh Mental Well-Being Scale (SWEMWBS) within adult community mental health teams. Measures were repeated 3 months later. Professionals recorded the setting, refusal rates and cluster diagnosis.

Results A total of 245 patients completed 674 measures, demonstrating good initial return rates (81\%), excellent scale completion (98-99\%) and infrequent refusal/unsuitability (11\%). Only $32(13 \%)$ returned follow-up measures. Significant improvements occurred in functioning $(P=0.01)$, PHQ-9 $(P=0.02)$ and GAD-7 $(P=0.003)$ scores (Cohen's $d=0.52-0.77)$ but not in SWEMWBS $(P=0.91)$ scores. Supercluster A had higher initial PHQ-9 and GAD-7 scores $(P<0.001)$ and lower SWEMWBS scores $(P=0.003)$ than supercluster $B$. Supercluster $C$ showed the greatest functional impairment $(P=0.003)$
\end{abstract}

Clinical implications PHQ-9 and GAD-7 appear acceptable as patient-reported outcome measures in community mental health team. SWEMWBS seems insensitive to change. National outcome programmes should ensure good follow-up rates.

Declaration of interest None.

Keywords Community mental health teams; patients; rating scales; outcome studies; out-patient treatment.
Reliable, valid and practical outcome measures are a priority for mental health services. ${ }^{1}$ It is now essential for clinical teams to report outcomes in order to evaluate their work, demonstrate effectiveness and support future commissioning decisions. ${ }^{2}$ However, few pragmatic studies exist to inform delivery of mental health outcomes programmes, ${ }^{3,4}$ including current initiatives within the UK National Health Service (NHS).

The NHS quality agenda promotes three central themes: effective services, safety and a positive patient experience. ${ }^{5}$ Arguably, it is the users of services who are best placed to judge how they feel. ${ }^{6}$ Patient-reported outcome measures (PROMs) are standardised questionnaires that elicit subjective reports of health and illness. They aim to assess the personal effects of symptoms, functioning, problems, risks and general well-being on an individual's quality of life. However, no single PROM has evidence of validity across all areas of mental health. ${ }^{7}$ Community mental health teams (CMHTs) are a key component of specialist mental healthcare, yet front-line use of PROMs has not been systematically evaluated in this setting. In addition, it remains unclear how outcomes in secondary care vary across mental healthcare clusters. 'Clustering' is an important tool within the National Tariff Payment System ('Payment by Results') and is recommended by NHS England in order to deliver its Five Year Forward View for Mental Health. ${ }^{9}$

\section{Aims of this study}

This study evaluated routine use of three PROMs within adult CMHTs: the Patient Health Questionnaire (PHQ-9), the Generalised Anxiety Disorder Scale (GAD-7) and the Short Warwick-Edinburgh Mental Well-Being Scale (SWEMWBS). We aimed to:

(a) assess completion rates and patient acceptability;

(b) evaluate responsiveness - comparing measures at initial assessment and at review/discharge across mental health superclusters

\section{Method}

This project was registered as a service evaluation by the Department of Research and Development at Leeds and York Partnership NHS Foundation Trust and granted NHS research governance approval (R\&D no: LYPFT 2014/498/L). 


\section{Setting and participants}

Secondary care mental health services in York and Selby are provided to a population of 280000 by a specialised mental health trust. The population is predominantly White British (95\%), with those of Asian ethnicity (2.2\%) representing the largest single ethnic minority. ${ }^{10}$ We collected data from May to October 2014 from the two large 'ageless' adult CMHTs. NHS data from trust informatics during the study showed that $38 \%$ of contacts were new referrals, with $31 \%$ of patients classified as being under the Care Programme Approach (CPA). A mean of $78 \%$ of the total caseload were being seen each month.

\section{Data collection}

We included patients aged 18 years and over attending CMHT appointments. Individuals were receiving care from one or more professionals at a psychiatric clinic, at a community mental health team base, at home or in another setting.

Patients were invited to complete the SWEMWBS, PHQ-9 and GAD-7 scales together. Measures were posted with the appointment letter to new referrals to CMHTs, with a request to hand them to the professional they saw. Staff also offered the measures at the initial appointment to individuals who had not completed them. Patients were asked to complete the measures again at follow-up 3 months later, or at discharge if sooner. Follow-up questionnaires were offered in person at the appointment by reception staff or the professional the individual saw. We introduced the study at a team business meeting and obtained staff agreement to participate before the start. In addition to verbal reminders at team meetings during the 6 month study period, we contacted staff individually by e-mail on two occasions (at 3 and 5 months) to remind them to collect follow-up questionnaires.

Patients could choose to complete the measures before, during or immediately after their appointment. Forms explicitly stated that if an individual did not feel like completing the questionnaires, they could decline and this would not affect their care. The questionnaires also informed patients that they could choose to receive this information in audio format (for example, as a CD) or in other languages, including via an interpreter.

Using a standardised pro forma attached to the measures, we asked staff to record details about the clinical setting, the reason for seeing the patient, and the main mental health problem (care cluster and diagnosis). To assess return rates accurately, at both initial and follow-up time points we specifically asked staff to return the pro forma even if an individual was unable (or declined) to complete measures. Staff also entered the responses into the computerised clinical record. Missing data were later accessed from this record.

\section{Outcome measures}

PHQ-9

PHQ-9 is a nine-item measure of depressive symptoms. ${ }^{11}$ Each item is rated using four ordinal response options
(0, not at all; 3 , nearly every day), giving a severity score between 0 and 27. PHQ-9 also rates difficulty in functioning. A score greater than 9 indicates clinically significant depression. The PHQ-9 is well validated against standard criteria, demonstrates sensitivity to change and is used in a variety of clinical settings. ${ }^{12,13}$

\section{GAD-7}

GAD-7 is a seven-item measure of anxiety symptoms. ${ }^{14}$ Each item is rated on the same four ordinal responses as the PHQ-9, giving a severity score between 0 and 21. A score above 7 is recommended to identify a likely anxiety disorder.

PHQ-9 and GAD-7 form part of the UK Department of Health's National Minimum Data Set. ${ }^{3}$ Their use is supported by the National Institute for Health and Care Excellence for assessing clinical progress in mental health services. ${ }^{1}$

\section{SWEMWBS}

SWEMWBS is a short version of a measure originally developed to monitor well-being in the general population and to evaluate policies addressing well-being. ${ }^{15,16}$ There are seven items, each with five response categories (1, none of the time; 5 , all of the time). The score range is 7-35 and higher scores indicate greater mental well-being. At the time of this study, the local NHS adopted SWEWWBS within the Regional Care Pathways and Packages Project, designed to implement Mental Health Payment by Results. SWEMWBS has been reported to have adequate internal consistency and reliability. ${ }^{17}$ It has not been systematically evaluated in mental health populations. The developers recommended that sensitivity to change be demonstrated before its introduction into clinical settings.

Prospectively, we also aimed to analyse responses to the following three key questions separately.

- Self-harm risk (PHQ-9 question 9): 'How often have you been bothered by thoughts of being better off dead or of hurting yourself in some way?' This question is of particular interest in clinical risk assessments.

- Functional impairment (additional tenth PHQ-9 question): 'How difficult have these problems made it for you to do your work, take care of things at home, or get along with other people?' This question is of practical importance and is independent of symptom scoring.

- Problem-solving ability (SWEMWBS question 4): 'How often over the past 2 weeks would you agree that "I've been dealing with problems well?"' An inability to solve problems is significantly associated with hopelessness and suicidal intent. ${ }^{18}$

\section{Data analysis}

Data were anonymised and analysed using IBM Statistical Package for Social Sciences for Windows, version $22 .{ }^{19} \mathrm{We}$ adjusted total PHQ-9, GAD-7 and SWEMWBS scores for individuals who omitted some replies, using syntax coding 
with the following formula:

$$
\begin{aligned}
\text { Corrected score }= & \left(\frac{\text { Total score }}{\text { No of questions answered }}\right) \\
& \times \text { Total no. of questions. }
\end{aligned}
$$

This is a recommended way of handling potential bias in the analysis due to missing items in questionnaires with no subscales. ${ }^{20,21}$ Results are quoted as percentages to the nearest whole number, with totals based on valid known responses. Non-parametric tests were applied to ordinal and continuous variables. We used Spearman's correlation coefficient for independent samples at baseline, Wilcoxon's signed rank $(z)$ test for paired data (initial versus final outcomes), and the Kruskal-Wallis $H$-test for differences between superclusters. ${ }^{22}$ Clinical effect sizes (Cohen's $d$ ) were calculated for reported changes in measures. ${ }^{23}$

\section{Results}

\section{Response rate}

Individuals returned 674 out of 831 questionnaires - a completion rate of $81 \%$. These comprised 277 sets of forms (Table 1). Initial forms were completed by 245 patients, with similar response rates (78-81\%) for each PROM. Follow-up forms were received from 32 (13\%) individuals. The mean time period between initial and follow-up forms was 74 (s.d. 58) days. There were high rates of scale completion - most respondents answered all questions on each form. Professionals completed their part of the initial forms in $55 \%$ (134) cases. Staff recorded that nine (7\%) patients declined to fill in the initial forms, and judged it inappropriate to offer forms in another five (4\%) cases. No patient was reported to have declined or been judged unsuitable to complete follow-up questionnaires.

\section{Patients and setting}

The mean age of patients was 47 years (range 18-93), including $127(60 \%)$ women. Initial forms were handed in at a clinic or CMHT base in $99(74 \%)$ cases, and at home or another place in $34(26 \%)$ cases. Most patients $(96,72 \%)$ completed forms alone, $16(12 \%)$ received assistance from carers or relatives, and seven $(5 \%)$ had assistance from staff. No patient asked to receive the questionnaires in an audio format or in another language.

\section{Professionals}

A mean of 27 sets of forms (range 1-50) were returned by 25 professionals. These included five community psychiatric nurses, five social workers, two psychologists, one occupational therapist and 12 psychiatrists (five working age adult consultants, two older age consultants, four core trainees and one higher trainee). Psychiatrists returned twice as many measures per professional (mean 36, 438 forms, 65\% of total) as other staff (mean 18, 236 forms, 35\%), general $z$-test $P<0.0001,95 \%$ confidence interval $61-68 \% .^{20}$ The reason recorded for completing initial forms was assessment in 77 (63\%) cases, review (including CPA review) in 39 (32\%) cases and discharge in three $(2 \%)$ cases.

\section{Diagnosis}

The main diagnosis according to the 10th edition of the International Classification of Diseases ${ }^{24}$ was available for 211 (86\%) of patients. Most common was depressive disorder (acute, recurrent or chronic) in $71(34 \%)$ cases, psychosis (including schizophrenia) in 28 (13\%) cases and bipolar disorder (including mania) in 26 (13\%) cases. Personality disorder comprised $16(8 \%)$ cases, anxiety disorder $14(7 \%)$, dementia $13(6 \%)$, adjustment disorder $12(6 \%)$, alcohol or drug dependence nine (4\%), post-traumatic stress disorder nine (4\%), obsessive-compulsive disorder five (2\%) and other diagnoses eight (3\%).

\section{Correlations between outcomes}

Table 2 describes the associations between measures (construct validity). PHQ-9 and GAD-7 scores were strongly correlated with each other at initial and final appointments. There was a moderate correlation between initial and final PHQ-9 scores, and between initial and final GAD-7 scores. SWEMWBS also showed a moderately strong correlation with concurrent PHQ-9 and GAD-7 scores. However, we found no significant correlation between final SWEMWBS and initial scores on any of the measures.

\section{Initial versus final scores}

Table 3 shows initial and final outcome scores for the paired data $(n=32)$. Applying Wilcoxon's signed rank test, PHQ-9 and GAD-7 scores were significantly lower at review, whereas SWEMWBS showed no significant change. For specific questions, patients' median ratings for thoughts of selfharm and also for their ability to function day to day improved significantly. Respondents' perceived ability to solve problems did not change significantly.

\begin{tabular}{|lcccc|}
\hline Table 1 & Completion rates for outcome measures & & \\
Measure & $\begin{array}{c}\text { Initial forms completed } \\
(n=245), \text { no. }(\%)\end{array}$ & $\begin{array}{c}\text { Final forms completed } \\
(n=32), \text { no }(\%)\end{array}$ & $\begin{array}{c}\text { No. of questions per } \\
\text { form }\end{array}$ & $\begin{array}{c}\text { Total no. questions answered per form, } \\
\text { mean (s.d.) }\end{array}$ \\
\hline SWEMWBS & $192(78)$ & $26(81)$ & 7 & $6.94(0.31)$ \\
\hline PHQ9 & $198(81)$ & $31(97)$ & $9(+1)$ & $8.88(0.45)$ \\
\hline GAD7 & $196(80)$ & $31(97)$ & 7 & $6.85(0.90)$ \\
\hline
\end{tabular}




\begin{tabular}{|c|c|c|c|c|c|c|}
\hline Measure & Initial SWEMWBS & Initial PHQ9 & Initial GAD7 & Final SWEMWBS & Final PHQ9 & Final GAD7 \\
\hline Initial SWEMWBS & - & $-0.77^{\star \star \star}$ & $-0.70^{\star \star \star}$ & 0.20 & $-0.45^{\star}$ & $-0.48^{\star}$ \\
\hline Initial PHQ9 & $-0.77^{\star \star \star}$ & - & $0.81^{\star \star \star}$ & -0.02 & $0.48^{\star \star}$ & $0.49^{\star \star}$ \\
\hline Initial GAD7 & $-0.70^{\star \star \star}$ & $0.81^{\star \star \star}$ & - & 0.09 & 0.31 & $0.52^{\star \star}$ \\
\hline Final SWEMWBS & 0.20 & -0.02 & 0.09 & - & $-0.56^{\star \star}$ & $-0.49^{\star \star}$ \\
\hline Final PHQ9 & $-0.45^{\star}$ & $0.48^{\star \star}$ & 0.31 & $-0.56^{\star \star}$ & - & $0.83^{\star \star \star}$ \\
\hline Final GAD7 & $-0.48^{\star}$ & $0.49^{\star \star}$ & $0.52^{\star \star}$ & $-0.49^{\star \star}$ & $0.83^{\star \star \star}$ & - \\
\hline
\end{tabular}

Values are Spearman's $r .{ }^{\star} P<0.05,{ }^{\star \star} P<0.01,{ }^{\star \star \star} P<0.001$.

The mean initial PHQ-9 score of 16.8 (s.d. 7.6) decreased on review to 12.6 (s.d. 8.6), representing a moderate effect size (Cohen's $d=0.52$ ) across the total sample. The mean initial GAD-7 score of 12.9 (s.d. 6.2) also improved at follow-up to 8.1 (s.d. 6.1), indicating a large effect size $(d=0.77)$.

To examine whether there was any selection bias in follow-up responses, we compared initial median scores for those who did $(n=32)$ and did not $(n=213)$ complete final measures. The Mann-Whitney $U$-test for independent samples showed no significant difference on the SWEMWBS $(P=0.91)$, PHQ-9 $(P=0.42)$ or GAD-7 $(P=0.78)$.

\section{Age, gender and time interval}

For both initial and final measures, older patients answered fewer questions on the PHQ-9 $(r=-0.52, P=0.002)$ and GAD-7 $(r=-0.31, P<0.001)$. Age was correlated positively with initial SWEMWBS score (Spearman's $r=0.36, P<0.001$ ) and negatively with initial PHQ-9 $(r=-0.15, P=0.04)$ and ability to function $(r=-0.17, P=0.03)$. There was no significant association between age and any final outcome (SWEMWBS, PHQ-9, GAD-7 or functioning). We found no significant association between gender and initial or final measures. The time period between completion of initial and final forms also showed no significant correlation with any initial or final outcome.

\section{Superclusters}

Tables 4 and 5 describe patients and their outcomes across the three supercluster categories. ${ }^{8}$ There were significant differences between PHQ-9, GAD-7 and SWEMWBS scores at initial but not final review. Individuals with non-psychotic disorders (supercluster A) had lower initial SWEMWBS scores $(P<0.001)$, and high levels of anxiety and depressive symptoms that improved at review. Respondents with psychosis (supercluster B) had the lowest PHQ-9 and GAD-7 scores $(P=0.003)$. Those with organic disorders (supercluster $\mathrm{C}$, mainly dementia or cognitive impairment) had the greatest difficulty in functioning $(P=0.003)$ based on the PHQ-9 functioning question). They also reported significant depression, anxiety and self-harm thoughts. Insufficient responses were received to calculate reliable final median outcome scores for supercluster C.

\section{Discussion}

This is the first study to examine the pragmatic integration of the PHQ-9, GAD-7 and SWEMWBS within routine CMHT practice. For these three PROMs, we found good initial return rates $(80 \%)$, excellent rates of scale completion (98-99\%) and low rates (11\%) of patient refusal or unsuitability. After 3 months, patients reported significant improvements in symptoms of depression and anxiety, self-

\begin{tabular}{|c|c|c|c|c|}
\hline Measure & Initial score, median (IQR) & $\begin{array}{l}\text { Final score, median } \\
\text { (IQR) }\end{array}$ & $\begin{array}{c}\text { Change in paired scores, median } \\
\text { (IQR) }\end{array}$ & $\begin{array}{l}\text { Wilcoxon's (z) test } \\
\text { P-value }\end{array}$ \\
\hline SWEMWBS & $17.0(13.0-22.75)$ & $16.5(13.0-24.25)$ & $-1.5(-6.25$ to +6.3$)$ & 0.91 \\
\hline PHQ9 & $19.6(11.0-23.0)$ & $12.0(6.75-20.75)$ & $-3.0(-9.75$ to +3.0$)$ & $0.02^{\star}$ \\
\hline GAD7 & $15.0(9.0-18.0)$ & $7.5(2.75-12.25)$ & $-2.0(-8.0$ to 0.0$)$ & $0.003^{\star \star}$ \\
\hline $\begin{array}{l}\text { Thoughts of self-harm or being } \\
\text { better off dead }\end{array}$ & $\begin{array}{c}1.0(0.0-3.0) \\
\text { On several days in past } 2 \\
\text { weeks }\end{array}$ & $\begin{array}{l}(0.0-2.0) \\
\text { Not at all }\end{array}$ & $0.0(-1.0$ to 0.0$)$ & $0.008^{\star \star}$ \\
\hline $\begin{array}{l}\text { Difficulty in functioning day to } \\
\text { day }\end{array}$ & $\begin{array}{l}2.0(1.0-3.0) \\
\text { Extremely difficult }\end{array}$ & $\begin{array}{c}1.0(0.0-2.25) \\
\text { Somewhat difficult }\end{array}$ & 0.0 (-1.0 to 0.0$)$ & $0.013^{\star \star}$ \\
\hline Ability to deal with problems & $\begin{array}{c}2.0(2.0-3.0) \\
\text { Rarely }\end{array}$ & $\begin{array}{l}3.0(1.0-4.0) \\
\text { Some of the time }\end{array}$ & $0.0(-1.0$ to +2.0$)$ & 0.80 \\
\hline
\end{tabular}

$\mathrm{IQR}$, interquartile range. ${ }^{\star} P<0.05,{ }^{\star \star} P<0.01$.

Text shows wording of median response. 


\begin{tabular}{|c|c|c|c|c|c|}
\hline Supercluster & No. (\%) & $\begin{array}{c}\text { Age } \\
\text { (years) }\end{array}$ & $\begin{array}{c}\text { Thoughts of self-harm or being } \\
\text { better off dead }\end{array}$ & $\begin{array}{l}\text { Difficulty in functioning } \\
\text { day to day }\end{array}$ & $\begin{array}{l}\text { Ability to deal with } \\
\text { problems }\end{array}$ \\
\hline $\begin{array}{l}\text { A. Non- } \\
\text { Psychotic }\end{array}$ & $144(68)$ & 45.0 & 2.0 & 2.0 & 2.0 \\
\hline B. Psychotic & $54(26)$ & 44.5 & 1.0 & 1.5 & 3.0 \\
\hline C. Organic & $13(6)$ & 84.0 & 3.0 & 3.0 & 3.0 \\
\hline$P$-value & & $<0.001^{\star \star \star}$ & $0.036^{\star}$ & $0.003^{\star \star}$ & $0.002^{\star \star}$ \\
\hline
\end{tabular}

Figures are corrected median scores.

$P$-values from Kruskal-Wallis $H$-test for differences between superclusters. ${ }^{\star} P<0.05,{ }^{\star \star} P<0.01,{ }^{\star \star \star} P<0.001$.

harm thoughts and functioning, but not in subjective wellbeing or perceived ability to handle problems.

It is important that outcomes are validated for the population in which they are used. Decreasing anxiety scores were observed across superclusters A and B. Building on research in other settings, ${ }^{25,26}$ our study provides new evidence that the GAD-7, like PHQ-9, is responsive to change in a community mental health population. For depressive symptoms, a drop of more than five PHQ-9 points is reported to indicate a significant and reliable clinical improvement. ${ }^{27} \mathrm{We}$ found an eight-point reduction in PHQ-9 scores in supercluster A, which includes those diagnosed with depressive disorder. This effect is similar in size to those observed in large randomised treatment trials for depression. ${ }^{28}$ These findings suggest that both PHQ-9 and GAD-7 might be adopted as PROMs within secondary mental healthcare in functional (non-dementia) populations.

This study has several limitations. Patients and professionals were not asked about their views on the usefulness of collecting these PROMS, or about possible harms. It is also uncertain whether professionals used the responses during their meetings with patients to improve the quality of care (rather than simply to measure it). Furthermore, we do not know the extent to which the improvements observed were due to professional interventions (including medication and psychosocial approaches) rather than the passage of time or regression to the mean.

An important finding is the low collection rate $(n=32$, $13 \%$ ) for follow-up measures in 'real world' clinical practice. Other mental health outcome studies have also recorded follow-up rates as low as $10-25 \%$, even after (as in our study) professionals are prompted. ${ }^{29,30}$ The difference between initial and final response rates might in part be linked to the number of requests to complete measures. For completion of initial measures, patients were asked both in writing (posted with the appointment letter) and again in person at the appointment. By contrast, collection of follow-up measures relied on staff remembering to ask patients to complete forms at face-to-face clinical review alone.

While the low final response rate limits some conclusions drawn, the outcome score changes observed may be generalisable to the wider patient population for several reasons. First, our analysis comparing initial median scores for completers versus non-completers showed no significant difference in either PHQ-9, GAD-7 or SWEMWBS. Second, response rates by gender ratio were similar at initial and final follow-up, and the time interval between initial and final measures showed no significant relation with any outcome. We have no evidence to support the idea that individuals who improved the most were more, or less, likely to complete final measures. This suggests that attrition bias at follow-up - due to variations between patients in symptoms, functioning or well-being - is less likely. Third, we observed a large (50-fold) variation in the collection of PROMS between professionals. For example, psychiatrists returned twice as many initial and follow-up questionnaires as other team members. In conclusion, it appears more likely that differences in staff engagement with the study, and inconsistent prompting of patients to complete measures (rather than patient characteristics) may account for the variations in return rates.

However, a good response rate remains central to the future success of PROMs., ${ }^{3,6,9}$ This may be improved in busy CMHTs by providing clinicians with adequate administrative time and support, and by implementing robust electronic collection systems. ${ }^{2,3}$ For example, patients could complete forms directly on an electronic tablet linked to their clinical record.

Consistent with previous research, individuals with psychosis rated their well-being on SWEMWBS higher

\begin{tabular}{|lcccccr|}
\hline \multicolumn{2}{|l}{ Table 5} & Mental health superclusters: initial and final outcome scores & & & \\
Supercluster & Initial SWEMWBS & Initial PHQ9 & Initial GAD7 & Final SWEMWBS & Final PHQ9 & Final GAD7 \\
\hline A. Non- psychotic & 15.0 & 20.125 & 15.0 & 17.0 & 12.0 & 8.0 \\
\hline B. Psychotic & 22.0 & 11.0 & 9.0 & 15.0 & 11.0 & 6.0 \\
\hline C. Organic & 24.5 & 24.0 & 17.0 & & & \\
\hline P-value & $<0.001^{\star \star \star}$ & $0.003^{\star \star}$ & $0.002^{\star \star}$ & 0.48 & 0.51 & 0.63 \\
\hline
\end{tabular}

Figures are corrected initial median scores.

$P$-values from Kruskal-Wallis $H$-test for differences between superclusters. ${ }^{\star \star} P<0.01,{ }^{\star \star \star} P<0.001$ 
than those with affective disorders. ${ }^{31}$ However, overall SWEMWBS scores did not change in this study, and there was no significant correlation between initial and follow-up SWEMWBS ratings. There are several possible explanations for this. First, subjective well-being could lag behind improvements in symptoms and functioning. Second, SWEMWBS includes questions about areas such as feeling useful and close to people, ${ }^{16}$ which could be measuring something different from other outcomes. Third, the psychometric properties of SWEMWBS may include lower internal reliability and less sensitivity to clinical change than other PROMs. Future research in this population could evaluate the responsiveness of SWEMWBS using methods such as the standardised response mean, ${ }^{32}$ which allows for improvement or worsening over time. Alternative well-being measures are currently being developed. Recovering Quality of Life (http://www.reqol.org.uk) is a new national well-being PROM commissioned by the UK Department of Health. Specifically designed to assess quality of life and recovery outcomes in adults with different mental health conditions, it has been tested in 6000 mental health patients. ${ }^{30}$ The brief version (ReQoL-10) is now freely available for clinical and research use in the UK NHS.

\section{Acknowledgements}

We thank Victoria Allgar, Senior Statistician at the Department of Health Sciences, York University, for her advice, and all NHS staff who participated in this study.

\section{About the authors}

Paul Blenkiron, MMedSc, FRCPsych, is a Consultant Psychiatrist in Adult Mental Health at Tees, Esk and Wear Valleys NHS Foundation Trust, and Honorary Professor at Hull York Medical School; Lucy Goldsmith, PhD, is a Research Assistant at the Population Health Research Institute, St George's, University of London, UK.

\section{References}

1 National Institute for Health and Care Excellence. Commissioning Stepped Care for People with Common Mental Health Disorders: Guide \& Bench Marking Tool CMG41. NICE, 2011 (http://www.nice.org.uk/usingguidance/commissioningguides/commonmentalhealthdisorderservices/commonmentalhealthdisorderservices.jsp).

2 Tadros G. Intelligent outcome measures in liaison psychiatry: essential even if not desirable. BJPsych Bull 2016; 40: 195-8.

3 Department of Health. The IAPT Data Handbook Version 2.0: Guidance on Recording and Monitoring Outcomes to Support Local Evidence-Based Practice. Department of Health, 2011 (http://www.iapt.nhs.uk/).

4 Department of Health. NHS Outcomes Framework: List of Outcomes and Indicators in the NHS Outcomes Framework for 2016-17. Department of Health, 2016 (https://www.gov.uk/government/publications/ nhs-outcomes-framework-2016-to-2017)

5 Department of Health. The Operating Framework for the NHS in England 2012/2013. Department of Health, 2011.

6 Devlin NJ, Appleby J. Getting the Most Out of PROMS: Putting Health Outcomes at the Heart of NHS Decision Making. The King's Fund, 2010 (https://www.kingsfund.org.uk/sites/files/kf/

Getting-the-most-out-of-PROMs-Nancy-Devlin-John-Appleby-KingsFund-March-2010.pdf)

7 Coulter A. Measuring what matters to patients. BMJ 2017; 356: j816.
8 Trevithick L, Painter J, Keown P. Mental health clustering and diagnosis in psychiatric in-patients. BJPsych Bull 2015; 39(3): 119-23.

9 NHS England. The Five Year Forward View for Mental Health. NHS England, 2016 (https://www.england.nhs.uk/wp-content/uploads/ 2016/07/fyfv-mh.pdf).

10 Vale of York Clinical Commissioning Group. Vale of York Public Health Report: Equality Strategy - Population and Health Inequalities Data. Vale of York Clinical Commissioning Group, 2013 (http://www.valeofyorkccg.nhs.uk/data/uploads/governing-body-papers/4-july-2013/ item-6-public-health-report.pdf).

11 Kroenke K, Spitzer RL, Williams JB. The PHQ-9: validity of a brief depression severity measure. J Gen Int Med 2001; 16: 606-13.

12 Horton M, Perry AE. Screening for depression in primary care: a Rasch analysis of the PHQ-9. BJPsych Bull 2016; 40: 237-43.

13 McMillan D, Gilbody S, Richards D. Defining successful treatment outcome in depression using the PHQ-9: a comparison of methods. J Affect Disord 2010; 127: 122-9.

14 Kroenke K, Spitzer RL, Williams JB, Monahan PO, Lowe B. Anxiety disorders in primary care: prevalence, impairment, comorbidity and detection. Ann Int Med 2007; 146: 317-25.

15 Tennant R, Hiller L, Fishwick R, Stephen P, Joseph S, Weich S, et al. The Warwick-Edinburgh Mental Well-being Scale (WEMWBS): development and UK validation. Health Qual Life Outcomes 2007; 5: 63.

16 Stewart-Brown S, Tennant A, Tennant R, Platt S, Parkinson J, Weich S. Internal construct validity of the Warwick-Edinburgh Mental Well-being Scale (WEMWBS): a Rasch analysis using data from the Scottish Health Education Population Survey. Health Qual Life Outcomes 2009; 7: 15-22.

17 Haver A, Akerjordet K, Caputi P, Furunes T, Magee C. Measuring mental well-being: A validation of the Short Warwick-Edinburgh Mental Well-Being Scale in Norwegian and Swedish. Scand J Public Health 2015; 43(7): 721-7.

18 Milnes D, Owens D, Blenkiron P. Problems reported by deliberate selfharm patients: perception, hopelessness and suicidal intent. $J$ Psychosom Res 2002; 53(3): 819-22.

19 IBM Corp. IBM SPSS Statistics for Windows, Version 22.0. IBM released 2013

20 Bell MA, Fairclough DL, Fiero $\mathrm{MH}$, Butow PN. Handling missing items in the Hospital Anxiety and Depression Scale (HADS): a simulation study. BMC Res Notes 2016; 9(1): 479

21 Fairclough DL, Cella DF. Functional Assessment of Cancer Therapy (FACT-G): non-response to individual questions. Qual Life Res 1996; 5 (3): 321-9.

22 Altman DG. Practical Statistics for Medical Research. Chapman and Hall, 1991.

23 Cohen J. Statistical Power Analysis for the Behavioral Sciences (2nd edn) Lawrence Earlbaum Associates, 1988.

24 World Health Organization. International Classification of Diseases (Vol. 10). WHO, 1992

25 Löwe B, Decker O, Müller S, Brähler E, Schellberg D, Wolfgang H, et al. Validation and standardization of the Generalized Anxiety Disorder Screener (GAD-7) in the general population. Med Care 2008; 46(3): 266-74.

26 Kertz S, Bigda-Peyton J, Bjorgvinsson T. Validity of the Generalised Anxiety Disorder-7 scale in an acute psychiatric sample. Clin Psychol Psychother 2013; 20(5): 456-64.

27 Richards DA, Borglin G. Implementation of psychological therapies for anxiety and depression in routine practice: two year prospective cohort study. J Affect Disord 2011; 133: 51-60

28 Gilbody S, Littlewood E, Hewitt C, Brierley G, Tharmanathan P, Araya R. Computerised cognitive behaviour therapy as treatment for depression in primary care (REEACT trial): large scale pragmatic randomised controlled trial. BMJ 2015; 351: h5627.

29 Timimi S. Children and young people's improving access to psychological therapies: inspiring innovation or more of the same? BJPsych Bull 2015; 39: 57-60. 

$\mathrm{E}$, et al. Recovering Quality of Life (ReQoL): a new generic self-reported outcome measure for use with people experiencing mental health difficulties. Br J Psychiatry 2018; 212(1): 42-9.

31 Blenkiron $\mathrm{P}$, Hammill CA. What determines patients' satisfaction with their mental health care and quality of life? Postgrad Med J 2003; 79 (932): $337-40$
32 Van Sonderen $\mathrm{E}$, Middel B. Statistical significant change versus relevant or important change in (quasi) experimental design: some conceptual and methodological problems in estimating magnitude of interventionrelated change in health services research. Int J Integr Care 2002; 2: e15.

\title{
Factors influencing use of community treatment orders and quality of care that people receive: results of a national survey in England and Wales
}

\author{
Harry Lei, ${ }^{1}$ Kirsten Barnicot, ${ }^{1}$ [D Emily Maynard ${ }^{2}$ Angela Etherington, ${ }^{2}$ Krysia Zalewska, ${ }^{2}$ \\ Alan Quirk, ${ }^{2}$ Rahil Sanatinia, ${ }^{1}$ Stephen J. Cooper, ${ }^{2}$ Mike J. Crawford ${ }^{2}$
}

BJPsych Bulletin (2019) 43, 227-235, doi:10.1192/bjb.2019.23

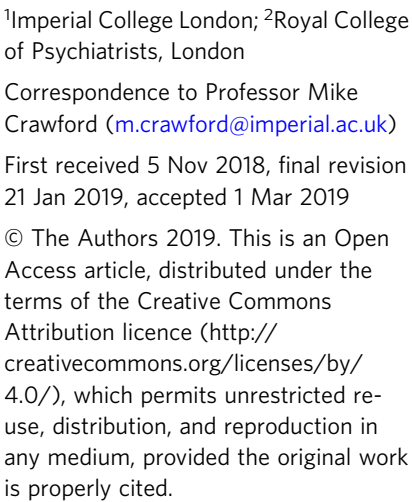

IImperial College London; ${ }^{2}$ Royal College of Psychiatrists, London

Correspondence to Professor Mike Crawford (m.crawford@imperial.ac.uk)

First received 5 Nov 2018, final revision 21 Jan 2019, accepted 1 Mar 2019

(c) The Authors 2019. This is an Open Access article, distributed under the terms of the Creative Commons Attribution licence (http:// creativecommons.org/licenses/by/ 4.0/), which permits unrestricted reuse, distribution, and reproduction in any medium, provided the original work is properly cited.

Aims and method We conducted a secondary analysis of data from the National Audit of Psychosis to identify factors associated with use of community treatment orders (CTOs) and assess the quality of care that people on CTOs receive.

Results Between 1.1 and $20.2 \%$ of patients in each trust were being treated on a CTO. Male gender, younger age, greater use of in-patient services, coexisting substance misuse and problems with cognition predicted use of CTOs. Patients on CTOs were more likely to be screened for physical health, have a current care plan, be given contact details for crisis support, and be offered cognitive-behavioural therapy.

Clinical implications CTOs appear to be used as a framework for delivering higher-quality care to people with more complex needs. High levels of variation in the use of CTOs indicate a need for better evidence about the effects of this approach to patient care.

\section{Declaration of interest None.}

Keywords Community treatment order; psychosis; community care; mental health act.
Community treatment orders (CTOs) were introduced in England and Wales in 2008, in an attempt to reduce the use of in-patient services for patients with poor adherence to their treatment. ${ }^{1-3}$ They require individuals with mental disorders who have been detained in hospital to adhere to treatment and supervision in the community. Non-compliance with CTOs may lead to revocation, where the patient is involuntarily admitted back into hospital for further treatment. The use of similar legislation to enforce community treatment also exists in other countries, including the USA, Australia and New Zealand. ${ }^{4,5}$

Although CTOs have been recommended as a method for improving adherence and patient safety, patients treated under CTOs feel more coerced. ${ }^{6}$ The use of CTOs has been much higher than was initially anticipated, ${ }^{7,8}$ and has increased considerably since they were first introduced. ${ }^{8,9}$ Concerns have been raised about greater use of compulsion amongst people from ethnic minority communities. ${ }^{10-12}$
Data submitted to NHS Digital in 2016 indicated that Black or Black British patients were almost nine times more likely to be treated on a CTO than White British patients. ${ }^{13}$ A systematic review of data from 38 studies of clinical practice in the UK, which compared the use of in-patient mental health services by different ethnic groups, found that Black patients were over four times more likely to be admitted to hospital on a compulsory basis than White patients. ${ }^{10}$ However, other studies have reported that associations between use of compulsory treatment and ethnicity may be reduced or eliminated when other sociodemographic and clinical factors are taken into account. ${ }^{14,15}$ To date, the influence of clinical and sociodemographic factors on the association between ethnicity and use of CTOs has not been examined.

There is considerable variation in the experiences of people treated under CTOs. ${ }^{6}$ Surveys of both patients and carers indicate that many believe their main aim is to try 